\title{
Ciencias sociales espacialmente integradas: la tendencia de Economía, Sociedad y Territorio ${ }^{1}$
}

\section{Introducción}

En décadas recientes el mundo científico ha visto emerger un nuevo campo del conocimiento: el análisis espaciotemporal. Esta nueva disciplina, compleja y necesariamente interdisciplinaria, incluye diversas áreas especializadas. No obstante, las que integran su plataforma fundamental son la estadística, la econometría espacial, los modelos de interacción espaciales; así como la informática y la programación, las altas matemáticas aplicadas, el manejo de sistemas de información geográfica y la geografía.

Cabe destacar que con frecuencia en la literatura internacional se habla de análisis espacial utilizando el término espacial como abreviación de espaciotemporal (lo mismo ocurre en este texto) (Anselin, 1995; Fotheringham et al., 1998; Granis, 2002). Los campos del conocimiento donde tiene aplicación el análisis espacial son muy diversos, tanto en las ciencias de la materia (física, química), como en las ciencias de la vida (salud, biología, ecología) y en las ciencias sociales y las humanidades (desarrollo urbano, economía, sociología, historia) (anexo 1).

La complejidad del análisis espacial y las múltiples habilidades que requiere han limitado su desarrollo en México, donde los trabajos que adoptan este tipo de análisis aún son excepciones. Esto no ocurre en la investigación que se realiza en los países desarrollados, donde la

\footnotetext{
${ }^{1}$ Este texto se deriva, en gran parte, de conversaciones personales con tres de los más destacados investigadores del mundo en análisis espacial aplicado a las ciencias sociales (también llamado análisis espacial o análisis espaciotemporal), que además son directores de prestigiados centros internacionales de investigación y desarrollo en esta nueva área del conocimiento: Luc Anselin (director del GeoDa Center for Geospatial Analysis and Computation, de la Arizona State University), Stewart Fotheringham (director del Centre for GeoInformatics de la Universidad de St. Andrews), y Michael Batty (director del Centre for Advanced Spatial Analysis de la Universidad de University College). Adicionalmente, debe destacarse el apoyo de diversos documentos del Center for Spatially Integrated Social Science (csiss), de la Universidad de California en Santa Bárbara, dirigido por Michael Goodchild.
} 
complejidad del análisis espacial se ha abordado mediante equipos de investigación integrados por especialistas diversos cuyos conocimientos y habilidades se complementan y potencian (Johnston et al., 2010).

Actualmente se acepta en la literatura internacional la notable superioridad del enfoque espaciotemporal sobre los enfoques cuantitativos y cualitativos tradicionales no-espaciales, en la investigación de múltiples temas clave de las ciencias sociales (y de las ciencias de la materia y de la vida). Muchos de los enfoques tradicionales no-espaciales (y no-temporales) están siendo seriamente cuestionados por su incapacidad para integrar (explícita y genuinamente) en sus marcos conceptuales y metodológicos el espacio y el tiempo (la localización absoluta y relativa de las variables que se analizan, así como el momento en que ocurren). La alternativa, entonces, es un enfoque de ciencias sociales espacialmente integradas, apoyadas sólidamente en el análisis espacial (Marcińczak, 2012).

El análisis espacial es complejo de explicar, pero se aborda más adelante (ver secciones 2-4). Por ahora simplemente se presenta un ejemplo deliberadamente sencillo con fines puramente ilustrativos: calcular un promedio simple se resuelve sumando los valores y dividiendo el total entre el número de observaciones, e.g. el promedio de tres calificaciones escolares: 4, 6 y $8=18 / 3=6$. Esto es correcto si la variable no tiene una localización espacial específica, como en el caso de las calificaciones que son no-espaciales (no tienen coordenadas geográficas).

Sin embargo, si la variable fuera un índice de desarrollo, cada variable tendría una localización en el territorio y estarían espacialmente relacionadas, es decir, no serían independientes entre sí en el territorio. Por lo tanto, no sería correcto estimar un promedio simple sino que se debería calcular un promedio espacial. En este caso cada valor de la variable analizada (calificaciones escolares: 4, 6, 8) tendría una localización concreta en el espacio (coordenadas: $x, y$ ) y temporal (fecha) que debe incluirse en el análisis, porque la gran mayoría de los procesos sociales tienen dimensiones espaciales y temporales, por lo que no ocurren en un no-lugar y en un no-tiempo. Al incluir la localización espacial y temporal los cálculos se complican notablemente (particularmente con grandes bases de datos), pero los resultados son mucho más poderosos para entender múltiples procesos sociales (Bailey y Gatrell, 1995). La literatura internacional sobre este tema ya es abundante.

\section{Los problemas clave de los análisis no-espaciales}

Los análisis no-espaciales registran, de inicio, las siguientes fallas fundamentales: 
a) A menudo generan los mismos resultados para patrones territoriales diferentes, porque no consideran la localización de los datos en el territorio. Es decir, analizan estadísticamente datos que ocurren en ningún lugar falla 1, llamada el "Problema del tablero de ajedrez": (White, 1983; Morrill, 1991). Esto se entiende mejor con un ejemplo: supongamos datos de segregación residencial organizados en una hoja de cálculo. Cada renglón contiene la información sociodemográfica de cada unidad espacial (colonia, AGEB) que integra una ciudad, pero no incluye la variable localización (coordenadas). Así las cosas, en la hoja de cálculo se pueden intercambiar renglones completos de información sociodemográfica entre las unidades espaciales, es decir, se puede alterar de múltiples maneras el patrón sociodemográfico al interior de la ciudad, y el resultado del análisis siempre será el mismo;

b) La estadística estándar no considera criterios de vecindad entre los datos (puntos o áreas), por lo que ignora preguntas clave como las siguientes: ¿Cómo se definen las unidades espaciales vecinas en una ciudad? ¿Son las que comparten un vértice por pequeño que sea, incluso un punto, el llamado "criterio del Alfil"? ¿Son las que comparten fronteras en alguna de las cuatro direcciones cardinales, el "criterio de la Torre"? ¿¿Son las que comparten tanto vértices como una frontera por lo menos, el "criterio de la Reina"? Aún más, ¿ Se podría definir la vecindad considerando los vecinos de los vecinos (los llamados vecinos de segundo orden)? ¿ O los vecinos de los vecinos de los vecinos... (los llamados vecinos de orden $k$ )? ¿Es posible definir unidades vecinas (o contiguas) estableciendo un cierto umbral de distancia (las localizadas a menos de " $\mathrm{x}$ " metros o kilómetros)? e incluso, ¿̨la distancia considerada en ese umbral podría estar afectada por un coeficiente de impedancia que reflejara la fricción de la distancia que podría expresarse mediante una función matemática más o menos compleja? Todos estos son criterios espaciales que, lamentablemente, la estadística estándar no-espacial no ubica ni remotamente en su radar. Esta falla 2 de la estadística estándar es conocida como "El problema de la definición de la vecindad" (Reardon y O'Sullivan, 2004; Garrocho y Campos, 2013).

c) Los resultados de la estadística estándar a menudo se representan en mapas, lo que genera la ilusión de estar trabajando con información espacial. Sin embargo, la interpretación de los resultados depende, al final, de la inspección visual de las representaciones 
cartográficas (mapas) para revelar lo que ocurre al interior de las zonas de estudio, lo que está expuesto a conocidas ilusiones ópticas $y$, en el extremo, provoca que "cada quién vea lo que quiere ver", falla 3, llamada el "Problema de la falta de indicadores locales", (Allen y Turner, 2005). Esto registra serios riesgos como lo ha demostrado desde hace décadas la sicología de la Gestalt (Metzeger, 2006).

d) Los resultados de la estadística estándar dependen enteramente de la manera de agrupar espacialmente los datos (la división artificial del espacio en AGEB, municipios y estados): En otras palabras el problema radica en la forma de dividir el territorio afecta directamente el entendimiento e interpretación de diversos fenómenos socioespaciales. Un ejemplo: imaginemos un espacio continuo como una mesa de billar en donde están distribuidas bolas rojas y blancas (personas con VIH y personas sanas). El análisis de esta distribución y su evolución en el tiempo generaría una cierta interpretación considerando ese espacio continuo. Pero si la mesa de billar se divide artificialmente en dos partes, dibujando una línea caprichosa que deja de un lado las bolas rojas y del otro las bolas blancas, y se suman los datos de cada área -es lo que usualmente hacen las oficinas recolectoras de datos al dividir artificialmente el espacio continuo en AGEB, municipios, estados-, nuestra interpretación de la distribución sería totalmente distinta: resultará una unidad espacial completamente sana y otra completamente afectada por el viH. Es claro que los resultados de análisis científicos no pueden depender de la manera como la oficina recolectora de información (INEGI) o los investigadores agregan espacialmente los datos, falla 4, el "Problema de la unidad espacial modificable" (Openshaw, 1984a).

e) Las unidades espaciales discretas, delimitadas artificialmente, se consideran homogéneas en su interior, lo que implica el grave "Problema de la falacia ecológica", falla 5 (Opensahaw, 1984b y Spicker, 2001). Esto se entiende mejor si se recupera el ejemplo del inciso anterior, en donde al dividir el espacio continuo con una línea caprichosa resultan dos unidades espaciales homogéneas en su interior. Esto también hubiera ocurrido si la línea divisoria del espacio continuo dejara una mezcla de bolas rojas y blancas en cada unidad espacial, porque al agregar la información para cada una de ellas el resultado sería un promedio que enmascararía las diferencias al interior de ambas zonas. 


\section{Las limitaciones técnicas de la estadística estándar (a-espacial) para analizar variables espaciales}

\subsection{El supuesto insostenible de la independencia espacial de las variables espaciales}

La estadística estándar a-espacial (incluyendo la econometría estándar) asume que las variables analizadas son independientes entre sí y estacionarias. Estos son supuestos fundamentales de la estadística no-espacial. Por independencia de los datos se entiende que no existe(n) relación(es) entre ellos o que no existe relación entre una misma serie de datos de una misma variable. Sin embargo, los datos espaciales registran al menos una relación inherente: dependencia espacial que se expresa en la cercanía o lejanía que existe entre su localización, que es producto de procesos diversos y complejos, o que puede ser simplemente aleatoria (Bailey y Gatrell, 1995).

La dependencia espacial (DE) se produce cuando el valor de una variable en una localización espacial es, aunque sea parcialmente, función del valor de la misma variable (o de otra variable) en unidades vecinas (áreas: como vecindarios, municipios, o puntos: como la representación de delitos en el territorio). Ejemplos de esta dependencia espacial de los datos existen en fenómenos como la difusión espacial de enfermedades, la aglomeración o rechazo entre ciertos negocios en las ciudades, la concentración territorial de población de bajo ingreso, el patrón locacional de crímenes violentos en el territorio o la segregación socioespacial de ciertos grupos de edad (Marcińczak, 2012).

Por su parte, por variables estacionarias se entiende que las propiedades estadísticas de las variables (los datos) no dependen de su localización en el espacio y en el tiempo. Sin embargo, este supuesto es insostenible cuando se habla de datos espaciales, porque las características clave de su distribución estadística (media, varianza o correlación espacial o temporal entre las variables) dependen necesariamente de su localización en el territorio (y en el tiempo). Cuando las variables no son estacionarias en el espacio se dice que registran heterogeneidad espacial (HE), lo que se refiere a que los valores de las variables dependen, aunque sea en parte, de su localización en el territorio. En síntesis: la heterogeneidad espacial de los datos se debe a una variación real y sustantiva asociada a su localización espaciotemporal, que evidencia la existencia y validez del contexto geográfico en el comportamiento de un fenómeno (Bailey y Gatrell, 1995).

Una seria implicación metodológica de ignorar la dependencia espacial en el análisis de regresión, por ejemplo, es que los coeficientes serán ineficientes (incluso espurios) para mostrar la relación entre variables. Por 
otro lado, la consecuencia de ignorar la heterogeneidad espacial es que, además del riesgo de obtener coeficientes ineficientes (espurios), las pruebas de significancia estadística también serán cuestionables debido a la inflación en los errores estándar. Estas son graves debilidades de la estadística estándar (no-espacial) cuando se manejan datos espaciales, como muchos que se analizan en las ciencias sociales. En síntesis, la falla central de la estadística no-espacial es no incluir explícitamente la localización en el territorio de las variables bajo estudio (longitud y latitud). Este problema lo corrige la estadística espacial.

\subsection{Las limitaciones del espacio discreto}

Desde una perspectiva espacial, vale reiterarlo, las metodologías estadísticas pueden dividirse en dos grandes categorías en términos de la manera como agrupan la información (los datos): las que consideran el territorio como un espacio discreto (dividido en unidades espaciales: vecindarios, AGEB, municipios, estados) y las que lo consideran como un espacio continuo (no dividido en unidades espaciales, sino como un espacio sin límites interiores). Las primeras han sido ampliamente utilizadas, pero se reconoce que tienen importantes limitaciones. Algunas de las principales son las siguientes: a) Se apoyan en datos artificialmente agregados en unidades espaciales discretas, es decir, en países, estados, municipios, AGEB, cuyos límites arbitrarios no corresponden necesariamente con el comportamiento de los fenómenos sociales bajo análisis; b) Las unidades espaciales discretas utilizadas se consideran homogéneas en su interior (lo que implica el grave problema de la falacia ecológica); c) La delimitación (forma) y escala (tamaño) de las unidades espaciales inciden directamente en los resultados de los análisis y, por tanto, en la manera como se entienden los fenómenos sociales bajo análisis, el "problema de la unidad espacial modificable" (Openshaw, 1984a).

Las debilidades de las metodologías basadas en la concepción del espacio discreto son adecuadamente subsanadas por las que lo conciben de manera continua. Las metodologías basadas en la concepción del espacio continuo se apoyan en complicados cálculos numéricos, por lo que requieren de software adecuado, que a menudo se tiene que desarrollar exprofeso (Anselin et al., 2006).

\subsection{El problema de la escala}

Con frecuencia los resultados de los análisis de fenómenos sociales dependen de la escala espacial a la que son observados: los procesos sociales que operan a diversos rangos de distancia (Tobler, 1970). Este aspecto 
clave no lo considera la estadística tradicional (no-espacial). Un ejemplo claro es la investigación de los patrones locacionales de las unidades económicas: pueden registrar patrones aglomerados a una cierta escala, patrones dispersos a otra y patrones aleatorios a una escala adicional. Lo que se requiere, entonces, es contar con metodologías multiescalares que permitan analizar fenómenos sociales a diversas escalas de manera simultánea (Sassen y Dotan, 2011). Esto no lo puede hacer la estadística tradicional (no-espacial), pero sí lo resuelve la estadística espacial.

\subsection{La importancia del movimiento y los fujos en el territorio}

Cuando se habla de movimiento y flujos ocurre con frecuencia que se vinculan estos temas con problemas de transporte. Sin embargo, en el análisis espaciotemporal esto va mucho más allá y se ocupa de múltiples tipos de flujos: innovaciones e ideas, remesas, financieros e información en internet, es decir, flujos intangibles; o bien flujos tangibles: compradores a unidades comerciales y de servicios, migrantes nacionales e internacionales, entre otros (Fotheringham, 1983).

$\mathrm{El}$ análisis espaciotemporal se enfoca en desarrollar nuevos métodos de análisis y construcción de modelos que permitan simular diversos tipos de flujos en el espacio y en el tiempo: entre países, regiones, ciudades y al interior de las ciudades. La mayoría de estos métodos se fundamentan en la teoría de interacción espacial, la cual explica las interacciones en el territorio: en modelos basados en agentes que simulan acciones e interacciones de agentes autónomos individuales o colectivos en modelos de autómatas celulares (estructuras de agentes comunicados entre sí de forma regular, de manera que el comportamiento de cada uno está afectado por el de sus vecinos), y en modelos de microsimulación (aplicaciones informáticas orientadas a simular el comportamiento en el territorio de unidades económicas a nivel micro, en especial hogares o individuos) (Batty, 2013). La estadística estándar a-espacial no tiene estos temas en su radar, pero son clave para los estudios urbanos y regionales.

\section{Espacio y tiempo: el reto de integrarlos a las ciencias sociales y al diseño de políticas públicas y privadas}

Otorgarle mayor importancia al espacio y al tiempo en la investigación en ciencias sociales podría acelerar notablemente su desarrollo e incrementar la aplicación de sus resultados al diseño de políticas públicas y privadas. Algunos elementos que respaldan esta aseveración son los siguientes: 
a) Los avances en las tecnologías de la información geográfica son sistemáticos y sorprendentes (velocidad de procesamiento informático, capacidad de almacenamiento y manejo de grandes bases de datos, nuevos instrumentos de análisis y visualización). En particular, los sistemas de información geográfica (SIG) posibilitan el almacenamiento, manejo y visualización de inmensas bases de datos espaciotemporales con una rapidez y facilidad inimaginables hace unos cuantos años, salvo en el plano teórico. Los SIG son al análisis espaciotemporal lo que el procesador de palabras a la escritura o las hojas de cálculo a los cálculos numéricos, sólo que manejan otro tipo de información. Es decir, los sIG son una herramienta indispensable para trabajar con bases de datos espaciales de grandes dimensiones y ordenar su integración, visualización y análisis desde niveles de resolución espacial de menos de un metro cuadrado hasta megaregiones continentales (Goodchild, 1992; Longley et al., 2008). Actualmente se imparten cursos de manejo de sIG en prácticamente todas las universidades de los países desarrollados y sólo en pocas instituciones de educación superior de México. Los avances tecnológicos abren nuevos y estimulantes horizontes de investigación conceptual y aplicada, vinculada al diseño de políticas públicas y privadas, que prometen empujar las fronteras del conocimiento en múltiples campos científicos, incluyendo las ciencias sociales (anexo 1).

b) Lo anterior se complementa de manera crucial con las nuevas fuentes de información disponibles en México, que registran estándares de calidad cada vez más altos. Estas fuentes de información no se podrán explotar adecuadamente si no se dominan nuevas técnicas de análisis espaciotemporal, e.g. el Directorio Estadístico Nacional de Unidades Económicas, que reporta las coordenadas precisas de la localización de todas las unidades económicas del país. La falta de capacidades analíticas espaciotemporales de gran parte de los profesionales de las ciencias sociales de México genera el alto riesgo de que estas nuevas bases de datos sean subutilizadas y, en consecuencia, se dejen de producir en el país.

c) En el pasado, y aún hoy, el análisis de datos espaciales se ha visto gravemente obstaculizado por las limitaciones de la estadística tradicional (la estadística no-espacial). En particular, por el hecho de que la información espaciotemporal viola algunos de sus supuestos básicos, especialmente la independencia y la no-heterogeneidad espacial de las variables bajo análisis. Sin embargo, recientemente 
los métodos para superar estos problemas estadísticos han avanzado de manera espectacular en un área del conocimiento relativamente nueva: el análisis espacial. Actualmente estos métodos estadísticos espaciales están listos para ser aplicados por los científicos sociales (Anselin et al., 2006; Anselin y Rey, 2014). No obstante, en nuestro país son pocos los que los conocen y menos los que están capacitados para utilizarlos (entre otros, destacan Vilalta, 2005 y 2010). De cualquier manera, debe quedar claro que a menudo es un grave error utilizar métodos de estadística no-espacial cuando se manejan datos espaciales, lo que lamentablemente es una práctica común en las ciencias sociales de México.

d) Es claro que el mundo registra importantes transformaciones que afectan profundamente la organización espacial, social, económica, política, ambiental y cultural de la población, a diferentes escalas geográficas (barrios, ciudades, regiones, países). Como esto incluye temas centrales de las ciencias sociales, se ha generado un enorme y creciente interés por incluir el espacio y el tiempo en sus entramados conceptuales y metodológicos (lo mismo ha ocurrido con muchas otras disciplinas ubicadas fuera de las ciencias sociales, ver anexo 1). Esto se refleja en los análisis cada vez más diversos de sistemas integrados por agentes numerosos, heterogéneos y complejos que interactúan en territorios concretos (Reardon y O'Sullivan, 2004). Usualmente el objetivo de estos trabajos es entender el comportamiento social (a diversos grados de agregación: desde el individual hasta el colectivo) y sus implicaciones en espacios específicos, para, cada vez con mayor frecuencia, apoyar el diseño de políticas públicas y privadas más focalizadas (planteadas a la medida).

e) El enfoque espaciotemporal ofrece la posibilidad de integrar los análisis de diversos procesos sociales. Si bien las tradiciones reduccionistas en la ciencia han establecido fronteras disciplinarias (a menudo arbitrarias) que conducen a realizar análisis sociales aislados unos de otros; en el mundo real, los procesos sociales interactúan dinámicamente entre sí, en entornos espaciotemporales específicos. El análisis espacial (que, como se ha subrayado, incluye la dimensión temporal) ofrece nuevos instrumentos y métodos para incorporar explícitamente el espacio y el tiempo a los análisis sociales, con el fin de lograr una mejor comprensión de diversos procesos y apoyar diseños más sólidos de políticas públicas y privadas con base científica. El espacio supera al tiempo como la estructura de 
integración de procesos sociales, pero la suma del espacio y el tiempo superan al espacio en solitario (Longley et al., 2008). ${ }^{2}$ Por ello el mejor enfoque integrador disponible por ahora para las ciencias sociales es el enfoque espaciotemporal.

f) Los análisis sociales aislados entre sí (no integrados en términos espaciotemporales) pueden generar conocimiento valioso, pero desvinculado de las circunstancias particulares de una cierta población en un espacio específico y en un determinado tiempo. Esto complica conectar la ciencia con el diseńo de políticas públicas y privadas. Un ejemplo que sucede con frecuencia: se generan políticas sociales que ignoran lo económico, o políticas económicas que ignoran lo ambiental, o políticas ambientales que ignoran lo social. El enfoque espaciotemporal reduce la complejidad de integrar análisis aislados, por lo que ofrece enormes oportunidades en términos científicos y prácticos, para generar políticas públicas espacialmente integradas (Garrocho et al., 2015).

\section{México: rezago en análisis espacial}

Mientras un grupo creciente de científicos sociales (y de otras áreas de la ciencia) en países avanzados están utilizando, innovando y desarrollando tecnologías sofisticadas y nuevos métodos de análisis (avances en sIG, modelos de simulación, desarrollo de software, análisis espacial: Anselin y Rey, 2014; Gelfand et al., 2010; Oyana y Margai, 2015, entre muchos otros), en algunos países emergentes, como México, se registra un rezago científico cada vez mayor. Los avances recientes en tecnología, teoría y metodología que apoyan el enfoque espaciotemporal podrían diseminarse sustancialmente en nuestro país para que cada vez más científicos sociales mexicanos los utilicen en sus trabajos de investigación. Es evidente que la incorporación del espacio y el tiempo a los análisis sociales no puede resolverse satisfactoriamente con los métodos e instrumentos convencionales que prevalecen en nuestro país (y prácticamente en toda latinoamerica).

Una revisión de la literatura identifica al menos 23 áreas del conocimiento que registran aplicaciones de análisis espaciotemporal, tanto en el mundo científico, como en el de los negocios y en el sector público (diseño, instrumentación, seguimiento y evaluación de políticas públicas). Estas 23 áreas del conocimiento son sólo una muestra derivada de la li-

\footnotetext{
${ }^{2}$ Es más productivo e interesante analizar simultáneamente diversos procesos en un mismo territorio en un cierto periodo, que los mismos procesos en un cierto periodo pero en cualquier lugar.
} 
teratura sobre análisis espacial. Se puede ver el listado de las áreas del conocimiento identificadas, con ejemplos de aplicación para cada una, en el anexo 1.

Una revista científica enfocada preferentemente, pero no solamente, en el análisis espacial podría potenciar esfuerzos para detonar las capacidades de los científicos sociales mexicanos, con el fin de aprovechar las inmensas posibilidades que ofrece el enfoque espaciotemporal al análisis social. Es decir, impulsar un enfoque explícitamente espaciotemporal cuyos métodos, instrumentos y resultados estén vinculados a la localización en el tiempo y en el espacio de las variables bajo estudio, lo que conduciría a considerar conceptos como distancia, localización, vecindad, adyacencia, dirección, conectividad, interacción espacial, procesos de aglomeración, dispersión o significancia y aleatoriedad, en términos de la estadística espacial. Esto podría ayudar a desarrollar nuevos métodos de análisis espaciotemporal y transformarlos en instrumentos amigables para los usuarios de las esferas científica, gubernamental y de los negocios; integrar los conceptos espaciotemporales a las teorías y prácticas de las ciencias sociales en México y diseminar el enfoque, instrumentos y métodos de análisis espaciotemporal.

Esto es lo que pretende Economía, Sociedad y Territorio. El enfoque espaciotemporal es de naturaleza ideográfica, no busca conocimiento generalizable (como las ciencias de la materia) sino entender procesos en ciertos tiempos y espacios de una manera replicable. Depende de un supuesto sencillo: cualquier modelo o teoría en las ciencias sociales es incapaz de explicar por completo los fenómenos que analiza y generalizar sus resultados, porque los procesos sociales son inherentemente heterogéneos en términos espaciales y temporales. Su interés, entonces, se enfoca en explicar las singularidades de procesos sociales en espacios y tiempos específicos más que a generar leyes universales causa-efecto.

\section{Anexo 1 \\ Áreas del conocimiento y algunos ejemplos de aplicaciones de análisis espaciotemporal}

En lo que sigue se registran sólo algunas de las múltiples aplicaciones de análisis espaciotemporal en diversas áreas del conocimiento, reportadas en la literatura internacional. Aunque se registran empalmes disciplinarios (inevitables y explicables), se debe tomar en cuenta que el eje transversal que articula todos los ejemplos de aplicación es su propósito de explicar estadísticamente los fenómenos bajo estudio, mediante métodos e instrumentos de análisis espaciotemporal. Esto, debido a las insalvables limitaciones técnicas y conceptuales de la estadística convencional (no-espacial) 
para analizar fenómenos espaciotemporales (ver las secciones iniciales de este documento, especialmente donde se analiza el problema de la independencia de las variables).

1.1. Geografía. Organización espacial de la economía, segregación residencial, crecimiento urbano, patrones locacionales de grupos de población (población mayor de 65 años), localización de empresas, accesibilidad a oportunidades de desarrollo (empleo, abasto, salud, educación y otros servicios fundamentales), patrones de desarrollo, estructura espacial del empleo y la población, desigualdades en materia de bienestar, patrones espaciales de la pobreza y de ganadores y perdedores de políticas públicas (población, ciudades y regiones), redes de ciudades, flujos de ideas, personas y mercancías, difusión en el uso de las Tic, disponibilidad de recursos naturales.

1.2. Estudios Urbanos. Eficiencia urbana, usos del suelo, expansión de la ciudad, conformación de mercados urbanos y regionales de suelo y vivienda, patrones espaciales de utilización, precio y plusvalía del suelo, perfil territorial de viviendas abandonadas o desocupadas, políticas habitacionales, así como su implementación y evaluación en el territorio, efectividad de políticas urbanas, modelos de simulación de la degradación de espacios intraurbanos.

1.3. Economía. Localización de la producción y vínculos en el espacio de los factores de la producción, patrones espaciales de innovaciones y patentes, diferencias de competitividad entre y al interior de las ciudades, conformación y coordinación de redes de negocios, localización intraurbana de actividades económicas de punta y alta dirección, econometría espacial, impactos de discontinuidades tecnológicas en ciudades y regiones.

1.4. Demografía. Flujos de migración interna e internacional, redes migratorias, patrones espaciales de defunciones por causa, de diferencias en las tasas de natalidad, de indicadores estandarizados de emigrantes e inmigrantes, estructura espacial del envejecimiento de la población a diferentes escalas geográficas, patrones geográficos de desempleo por grupos de edad, flujos de remesas, crecimiento demográfico diferenciado en el territorio.

1.5. Políticas Públicas. Localización espacial y sectorial de necesidades y expectativas sociales, escala de las inversiones públicas, 
diseño de políticas focalizadas dinámicas (de acuerdo a la situación y evolución de diversos factores), geografía de las finanzas públicas, patrones y difusión de necesidades y aspiraciones sociales en el tiempo y en el espacio, localización, magnitud y evolución espaciotemporal de grupos de población objetivo, evaluación de resultados de políticas públicas en regiones, ciudades y espacios intraurbanos, accesibilidad espacial a oportunidades de desarrollo, i.e. empleo, abasto, salud, educación, justicia, seguridad, patrones espaciales de bienestar y desarrollo humano, construcción de indicadores de desarrollo humano que integren variables espaciales fundamentales (acceso a oportunidades de desarrollo fundamentales).

1.6. Sociología. Distribución espacial de desigualdades sociales y económicas, concentraciones de pobreza en el espacio y en el tiempo (hyperghettos), delimitación de espacios de vida, conformación y evolución de redes de agentes sociales en el territorio, cambios de estilos de vida en regiones, ciudades y espacios intraurbanos, difusión de formas de pensar (ideologías, valores, percepciones, actitudes) y de modalidades de organización social, significado simbólico del espacio.

1.7. Antropología. Significado simbólico del espacio como estructurador de las actividades sociales, expansión y contracción espaciotemporal de ciertas manifestaciones culturales (grafiti, formas de lenguaje) o nacionalismos étnicos o identidades culturales, localización y utilización (desde lo local hasta lo internacional) de sitios simbólicos religiosos o históricos, comprobación empírica de teorías altamente cualitativas, alcance espacial de organizaciones a escala de comunidad o espacio intraurbano (barrio), patrones espaciotemporales de interconexiones transnacionales (mediante internet y envío de capital social: fotografías, videos), efectos socioespaciales de las urbanizaciones cerradas, estructura espacial del capital social y cultural, espacios cotidianos de las mujeres, movilidad espaciotemporal con enfoque de género.

1.8. Ciencia Política. Organización de los gobiernos y organizaciones de poder en el territorio, comportamiento espacial del voto, difusión de movimientos sociales en el tiempo y en el espacio, relación entre el poder y la conformación del espacio urbano, redes de poder en el territorio, comportamiento espacial de las mani- 
festaciones políticas, patrones espaciales de aceptación de diversos agentes políticos, localización de focos de descontento social, áreas prioritarias para realizar trabajo de concertación política.

1.9. Transporte. Modelos de transporte terrestre, aéreo y marítimo, estudios de movilidad en ciudades y regiones, accidentalidad en carreteras y calles, modelos integrados de usos de suelo y transporte para pronosticar el crecimiento urbano y las demandas de transporte, modelos de interacción espacial para simular flujos de vehículos o personas a diversas escalas espaciales: desde la nacional, hasta la de una estación de metro.

1.10. Ecología. Análisis de sostenibilidad ambiental y cambio climático, modelos dinámicos de relaciones entre variables socioeconómicas y variables medioambientales, modelos de pronósticos de contaminación, procesos de difusión o contracción de especies animales y vegetales, patrones espaciales de contaminación de agua, aire y suelos, identificación de zonas de riesgo natural o por la acción humana.

1.11. Salud Pública. Flujos cruzados de virus a diversas escalas espaciales (i.e. desde lo local hasta lo internacional: recordar el virus AH1N1), procesos espaciales de difusión de enfermedades (la difusión de viH en el distrito Castro en San Francisco o entre Estados Unidos y México), relación entre perfiles epidemiológicos con las características demográficas o del medioambiente o los efectos del cambio climático, localización eficiente de unidades de salud de todos los niveles de atención, rutas más eficientes de servicios móviles de salud, estructuras socioespaciales de utilización de los servicios de salud por grupos demográficos (nivel educativo, edad, género, religión), desigualdades de accesibilidad y utilización de los recursos de salud, cobertura efectiva de los servicios de salud, organización espacial de los servicios de salud privados, diferencias espaciotemporales entre los perfiles epidemiológicos de diferentes grupos de población (por niveles de ingreso, urbanos o rurales), patrones espaciales de prácticas sanitarias (hervir agua, lavarse las manos).

1.12. Sicología social. Percepción del espacio, la distancia, la ciudad y de diversos fenómenos socioespaciales por parte de diferentes grupos de población, factores determinantes de la subjetividad individual y colectiva en espacios intraurbanos, patrón espacial 
de la imagen de la inseguridad y el crimen, conducta espacial de la población en situaciones diversas (en situación de compras, en situación de emergencia), significado emocional del espacio, exposición y reacción a estímulos en el espacio urbano, territorios de acción y espacios de vida de la población por grupos de edad, ingreso, género.

1.13. Investigación educativa. Procesos y patrones espaciales de rendimiento escolar (en escuelas públicas y privadas), localización eficiente de escuelas de todos los niveles, desigualdades de acceso y utilización de los recursos educativos, cobertura efectiva de los servicios de todos los niveles, factores clave de la localización de los servicios educativos privados.

1.14. Criminología. Patrones del crimen a diversas escalas espaciales, difusión o contracción espaciotemporal del crimen, explicaciones espaciotemporales de los patrones de crimen, distribución espacial eficiente de los recursos anticrimen, diseño de rutas más eficaces de vigilancia y patrullaje, delimitación de espacios peligrosos (con diferentes intensidades de prioridad) a escala regional e intraurbana, estructura espacial de giros comerciales de alto riesgo en la ciudad (los llamados giros negros), factores sicológicos que crean y recrean problemas sociales en la ciudad, evaluación de las acciones anticrimen en el tiempo y en el espacio, identificación de factores clave que afectan los resultados de las políticas contra el crimen.

1.15. Historia. Alcance espacial de procesos históricos, difusión espacial de innovaciones a lo largo de la historia, flujos de comercio, dirección de la transmisión de avances en agricultura y ganadería, manejo de los materiales y la tecnología bélica (arco y flecha, ballesta, organización militar), disponibilidad de recursos lacustres y su evolución espaciotemporal, localización de recursos y barreras que impiden o favorecen la interacción y la difusión de ideas, organización administrativa de los gobiernos en el territorio, patrones de desarrollo social, económico, cultural de largo plazo, expansión del poder y de las naciones.

1.16. Arqueología. Procesos de difusión espacial de diversos tipos de herramientas o cerámicas y de la tecnología necesaria para su producción, organización espacial de espacios mortuorios, localización espaciotemporal de centros ceremoniales, patrones de 
dispersión de restos orgánicos, proximidad de diversas acciones sociales, relaciones espaciotemporales entre la producción y reproducción de acciones sociales, efectos espaciotemporales en procesos sociales de largo plazo (efectos de proximidad y lejanía: accesibilidad), relaciones de dependencia espaciotemporal en las acciones sociales, elementos clave que inciden la localización espacial y temporal de características humanas y físicas.

1.17. Estadística Espacial. Nuevos métodos econométricos, minimización del problema de la unidad espacial modificable, integración más transparente entre variables espaciales y temporales, construcción de modelos espaciotemporales (dinámicos y estocásticos) más robustos para explorar correlaciones, extrapolar datos y perfilar escenarios geoestadísticos, desarrollo de métodos más confiables para la identificación de clusters, mejores análisis multiescalares, métodos más sólidos y eficientes para incrementar la confiabilidad de los análisis geoestadísticos, manejo de grandes bancos de datos.

1.18. Matemáticas. Incorporación de la teoría del caos al análisis de fenómenos espaciotemporales donde el objetivo es entender y modelar la complejidad, coincidencia y simultaneidad de múltiples elementos en la dinámica de fenómenos y procesos no lineales ni predecibles sino azarosos o aleatorios (como muchos fenómenos sociales: las ciudades), desarrollo de nuevos métodos exploratorios y explicativos no lineales aplicables a diferentes escalas espaciales, a sistemas abiertos multilineales dinámicos y a diferentes fenómenos sociales en el tiempo y en el espacio, diseño de nuevos esquemas de interpretación epistémica y extrapolaciones de este nuevo conocimiento a la explicación de fenómenos espaciotemporales, mejor aprovechamiento de la geografía fractal para identificar regularidades espaciales de fenómenos sociales (más allá de la influencia de la escala y las singularidades locales), mejor simulación de escenarios complejos.

1.19. Ciencias Computacionales. Desarrollo de software en lenguajes libres, aplicaciones para lograr sistemas de información geográfica más poderosos y amigables, avances en geoinformática (o geocomputación), programación más eficiente de modelos espaciales dinámicos y estocásticos para simular escenarios en diversas áreas del conocimiento, mejor manejo, visualización y transmisión de grandes bases de datos. 
1.20. Comunicaciones. Nuevos métodos para capturar, almacenar, manejar, compartir, transferir, analizar y visualizar rápidamente grandes bases de datos espaciotemporales vía nuevas tecnologías de la información y las comunicaciones (TIC).

1.21. Mercadotecnia. Estudios de geomarketing, patrones espaciales de flujos de compradores en la ciudad, es decir,en el Centro Tradicional de Negocios y en espacios planificados tales como centros o plazas comerciales, patrones espaciales de tasas de ganancias potenciales, oportunidades de negocios en el espacio urbano, localización de negocios en entornos dinámicos y de alta competencia, alcance espacial de bienes, servicios y negocios (áreas de mercado).

1.22. Logística. Organización espaciotemporal de los recursos de las organizaciones y de los flujos de recursos, mercancías, energía e información en el espacio y en el tiempo, localización de unidades de almacenamiento, vínculos espaciotemporales entre la producción y los mercados, análisis espaciotemporales de gestión y planificación de compras, producción, transporte, almacenaje, manutención y distribución.

1.23. Oncología. Mediciones estadísticas de aglomeración, dispersión o aleatoriedad de células cancerosas para determinar la existencia de tumores o el inicio de procesos de metástasis.

Carlos Garrocho octubre, 2015

\section{Fuentes consultadas}

Allen, James P. y Eugene Turner (2005), "Ethnic residential concentrations in United States metropolitan areas", Geographical Review, 95 (2), American Geographical Society, New York, pp. 267-285.

Anselin, Luc (1995), "Local indicators of spatial association LISA", Geographical Analysis, 27 (2), Wiley Online Library, pp. 93-115.

Anselin, Luc, Ibnu Syabri y Youngihn Kho (2006), GeoDa: an introduction to spatial data analysis", Geographical Analysis, 38 (1), Wiley Online Library, pp. 5-22. 
Anselin, Luc y Sergio Rey (2014), Modern spatial econometrics in practice: a guide to GeoDa, GeoDaSpace and PySAL, GeoDa Press, University Phoenix, Arizona.

Bailey, C. Trevor y Gatrell, Anthony C. (1995), Interactive spatial data analysis, Essex-Longman, London.

Batty, Michael (2013), The new science of cities, MIt Press, Cambridge.

Fotheringham, A. Stewart (1983), "A new set of spatial-interaction models: the theory of competing destinations", Environment and Planning $A, 15$ (1), Sage Publications, pp. 15-36.

Fotheringham, A. Stewart, Martin Charlton y Chris Brunsdon (1998), "Geographically weighted regression: a natural evolution of the expansion method for spatial data analysis", Environment and Planning A, 30 (11), Sage Publications, pp. 1905-1927.

Garrocho, Carlos, Guillermo Aguilar, Carlos Brambila, Boris Graizbord y Jaime Sobrino (2015), Ciudades sostenibles en México: posicionamiento conceptual y operativo, UNFPA-Conapo, México.

Garrocho, Carlos y Juan Campos (2013) "Réquiem por los indicadores no espaciales de segregación residencial", Papeles de Población, 19 (77), Universidad Autónoma del Estado de México, Toluca, pp. 269-300.

Gelfand, Alan E., Peter Diggle, Peter Guttor y Montserrat Fuentes (eds.) (2010), Handbook of spatial statistics, Chapman \& Hall/CRC Handbooks of Modern Statistical Methods, CRC Press, Florida.

Goodchild, Michael F. (1992), "Geographical information science", International journal of geographical information systems, 6 (1), Taylor Francis Online, pp. 31-45.

Granis, Rick (2002), "Discussion: segregation indices and their functional inputs”, Sociological Methodology, 32 (1), Sage Journals, pp. 69-84.

Johnston, Ron; Michael Poulsen, y James Forrest (2010), "Evaluating changing residential segregation in Auckland", Using Spatial Statistics, 102 (1), Wiley Online Library, pp. 1-23. 
Longley, Paul A., Michael F. Goodchild, David J. Maguire y David W. Rhind (2008), Geographic information science and systems, John Wiley \& Sons, London.

Marcińczak, Szymon (2012), "The evolution of spatial patterns of residential segregation in Central European Cities: The Łódź Functional Urban Region from mature socialism to mature post-socialism”, Cities, 29 (5), Elsevier, pp. 300-309.

Metzeger, Wolfang (2006), Laws of seeing, MIT, Cambridge.

Morrill, Richard L. (1991), "On the measure of geographical segregation", Geography Research Forum, 11 (1), University of the Negev, Beerseba, pp. 25-36.

Openshaw, Stanley (1984a), "The modifiable spatial unit problem, concepts and techniques", Modern Geography, núm. 38, Geo Books, Norwich, pp. 1-40.

Openshaw, Stanley (1984b), "Ecological fallacies and the analysis of areal census data", Environment and Planning A, 16 (1), sage Publications, pp. 17-31.

Oyana, Tonny y Florence Margai (2015), Spatial analysis: statistics, visualization, and computational methods, CRC Press, Florida.

Reardon, Sean F. y David O'Sullivan (2004), "Measures of spatial segregation, sociological methodology", Sociological Methodology, 34 (1), Wiley Online Library, pp. 121-162.

Sassen, Saskia y Natan Dotan (2011), "Delegating, not returning, to the biosphere: How to use the multi-scalar and ecological properties of cities", Global Environmental Change, 21 (3), Elsevier, pp. 823-834.

Spicker, Paul (2001), "Poor areas and the 'ecological fallacy", Radical Statistics, vol. 76, Radical Statics Group, pp. 38-49.

Tobler, Waldo R. (1970), "A computer movie simulating urban growth in the Detroit region", Economic geography, Wiley Online Library, pp. 234-240. 
Vilalta, Carlos Javier (2005), "Cómo enseñar autocorrelación espacial", Economía, Sociedad y Territorio, 5 (18), Colegio Mexiquense A. C., Zinacantepec, pp. 323-333.

Vilalta, Carlos Javier (2010), “The spatial dynamics and socioeconomic correlates of drug arrests in Mexico city", Applied Geography, 30 (2), Elsevier, pp. 263-270.

White, Michael J. (1983), “The measurement of spatial segregation”, American Journal of Sociology, 88 (5), University of Chicago Press, Chicago, pp. 1008-1018. 